\title{
POSITIVE SOLUTIONS OF FOURTH-ORDER BOUNDARY VALUE PROBLEM WITH VARIABLE PARAMETERS
}

\author{
XIN DONG ${ }^{1}$ AND ZHANBING BAI ${ }^{2 *}$
}

Abstract. By means of calculation of the fixed point index in cone we consider the existence of one or two positive solutions for the fourth-order boundary value problem with variable parameters

$$
\left\{\begin{array}{l}
u^{(4)}(t)+B(t) u^{\prime \prime}(t)-A(t) u(t)=f\left(t, u(t), u^{\prime \prime}(t)\right), 0<t<1 \\
u(0)=u(1)=u^{\prime \prime}(0)=u^{\prime \prime}(1)=0
\end{array}\right.
$$

where $A(t), B(t) \in C[0,1]$ and $f(t, u, v):[0,1] \times[0, \infty) \times R \rightarrow[0, \infty)$ is continuous.

\section{INTRODUCTION AND PRELIMINARIES}

The deformation of an elastic beam in equilibrium state, whose two ends are simply supported, can be described by the fourth-order ordinary equation boundary value problem (BVP). Owning to its significance in physical, biological and chemical phenomena, the existence of positive solution for this problem has been studied by many authors. For example, some authors studied by the method of upper and lower solutions $[2,4,7]$, some studied by the fixed point index theorem $[9,10]$.

In 2003, Li [8] investigated the existence of positive solutions for fourth-order BVP with two parameters

$$
\left\{\begin{array}{l}
u^{(4)}(t)+\beta u^{\prime \prime}(t)-\alpha u(t)=f(t, u(t)), 0<t<1 \\
u(0)=u(1)=u^{\prime \prime}(0)=u^{\prime \prime}(1)=0
\end{array}\right.
$$

under the assumptions:

$(\mathrm{J} 1) f(t, u):[0,1] \times[0,+\infty) \rightarrow[0,+\infty)$ is continuous;

Date: Received: 2 March 2006; Revised: 15 August 15.

* Corresponding author.

2000 Mathematics Subject Classification. Primary 34B15; Secondary 34B18.

Key words and phrases. Boundary value problem, positive solution, fixed point, cone. 
(J2) $\alpha, \beta \in R$ and $\beta<2 \pi^{2}, \alpha \geq-\beta^{2} / 4, \alpha / \pi^{4}+\beta / \pi^{2}<1$.

Recently, Chai [5] studied the generalizing form as follows:

$$
\left\{\begin{array}{l}
u^{(4)}(t)+B(t) u^{\prime \prime}(t)-A(t) u(t)=f(t, u(t)), 0<t<1 \\
u(0)=u(1)=u^{\prime \prime}(0)=u^{\prime \prime}(1)=0
\end{array}\right.
$$

where $A(t)$ is non-negative.

In this paper, we concerned the following fourth-order boundary value problem

$$
\left\{\begin{array}{l}
u^{(4)}(t)+B(t) u^{\prime \prime}(t)-A(t) u(t)=f\left(t, u(t), u^{\prime \prime}(t)\right), 0<t<1 \\
u(0)=u(1)=u^{\prime \prime}(0)=u^{\prime \prime}(1)=0
\end{array}\right.
$$

Assume the following condition hold:

(A1) $f(t, u, v):[0,1] \times[0,+\infty) \times R \rightarrow[0,+\infty)$ is continuous;

(A2) $A(t), B(t) \in C[0,1], \alpha=i n f_{t \in[0,1]} A(t), \beta=i n f_{t \in[0,1]} B(t), \beta<2 \pi^{2}, \alpha \geq$ $-\frac{\beta^{2}}{4}, \frac{\alpha}{\pi^{4}}+\frac{\beta}{\pi^{2}}<1$.

where $A(t)$ can take negative values and the nonlinear function has the bending term.

This paper is organized as follows. In section 1 we give the introduction and some lemmas which needed in the proof of main results; Section 2 contain results for one or two positive solutions of the $\operatorname{BVP}(1.3)$.

Let $Y=C[0,1], Y_{+}=\{u \in Y: u(t) \geq 0, t \in[0,1]\}$. Obviously, $\left(Y,\|u\|_{0}\right)$ is Banach space, where $\|u\|_{0}=\sup _{t \in[0,1]}|u(t)|, u \in Y$. Setting $X=\left\{u \in C^{2}[0,1]\right.$ : $u(0)=u(1)=0\},\|u\|_{1}=\max \left\{\|u\|_{0},\left\|u^{\prime \prime}\right\|_{0}\right\}$, then $\left(X,\|u\|_{1}\right)$ is also Banach space. If $u \in C^{2}[0,1] \cap C^{4}(0,1)$ satisfies $\operatorname{BVP}(1.3)$ and $u(t) \geq 0, t \in[0,1]$, then we call $u$ is the positive solution of $\operatorname{BVP}(1.3)$.

Lemma 1.1. ([5]) $\forall u \in X,\|u\|_{0} \leq\left\|u{ }^{\prime \prime}\right\|_{0}$.

Given $h \in Y$, consider the following BVP:

$$
\left\{\begin{array}{l}
u^{(4)}(t)+\beta u^{\prime \prime}(t)-\alpha u(t)=h(t), 0<t<1 \\
u(0)=u(1)=u^{\prime \prime}(0)=u^{\prime \prime}(1)=0
\end{array}\right.
$$

where $\alpha, \beta$ such that the condition $(A 2)$.

Obviously, the equation $P(\lambda) \triangleq \lambda^{2}+\beta \lambda-\alpha=0$ has two real solutions $\lambda_{1}, \lambda_{2}=$ $\frac{-\beta \pm \sqrt{\beta^{2}+4 \alpha}}{2}$, owning to $(A 2)$, we can get $\lambda_{1}>\lambda_{2}>-\pi^{2}$.

We assume that $G_{i}(t, s)(i=1,2)$ is the Green's function of the following boundary value problem:

$$
-u^{\prime \prime}(t)+\lambda_{i} u(t)=0, u(0)=u(1)=0 .
$$

We also need some other lemmas as follows:

Lemma 1.2. ([8]) $G_{i}(t, s)(i=1,2)$ has some properties as follows:

(i) $G_{i}(t, s)>0, \forall t, s \in(0,1)$;

(ii) $G_{i}(t, s) \leq C_{i} G_{i}(s, s), \forall t, s \in[0,1]$;

(iii) $G_{i}(t, s) \geq \delta_{i} G_{i}(t, t) G_{i}(s, s), \forall t, s \in[0,1]$.

where, if $\lambda_{i}>0, C_{i}=1, \delta_{i}=\frac{\omega_{i}}{\sinh \omega_{i}}$; if $\lambda_{i}=0, C_{i}=1, \delta_{i}=1$; if $-\pi^{2}<\lambda_{i}<$ $0, C_{i}=\frac{1}{\sin \omega_{i}}, \delta_{i}=\omega_{i} \sin \omega_{i}$ 
Lemma 1.3. ([5]) Let $K_{i}(t)=\int_{0}^{1} G_{i}(t, s) d s, t \in[0,1]$ and $D_{i}=\max _{t \in[0,1]} K_{i}(t),(i=$ $1,2)$, then $D_{i}=K_{i}\left(\frac{1}{2}\right)>0,(i=1,2)$ and satisfies

(i) If $\lambda_{i}>0, D_{i}=\frac{1}{\lambda_{i}}\left(1-\frac{1}{\cosh \frac{\omega_{i}}{2}}\right)$,

(ii) If $\lambda_{i}=0, D_{i}=\frac{1}{8}$,

(iii) If $-\pi^{2}<\lambda_{i}<0, D_{i}=\frac{1}{\lambda_{i}}\left(1-\frac{1}{\cos \frac{\omega_{i}}{2}}\right)$.

For any $h \in Y$, the linear $\operatorname{BVP}(1.4)$ has a unique solution $u$ which is denoted by $T h=u$, the operator $T$ can be expressed by

$$
(T h)(t)=\int_{0}^{1} \int_{0}^{1} G_{1}(t, s) G_{2}(s, \tau) h(\tau) d \tau d s .
$$

Lemma 1.4. $T: Y \rightarrow\left(X,\|u\|_{1}\right)$ is linear completely continuous, and $\|T\| \leq M$, where $M=\max \left\{\left(\left|\lambda_{1}\right| D_{1} D_{2}+D_{2}\right),\left(\left|\lambda_{2}\right| D_{1} D_{2}+D_{1}\right)\right\}$.

Proof. By the definition of $T$ we known that $T: Y \rightarrow\left(X,\|u\|_{1}\right)$ is linear completely continuous, so we only need to prove $\|T\| \leq M$.

For $\forall h \in Y, u=T h \in X, u(0)=u(1)=u^{\prime \prime}(0)=u^{\prime \prime}(1)=0$, setting $v=$ $-u^{\prime \prime}+\lambda_{2} u$, then $v(0)=v(1)=0$. By (1.5) and (1.6), we get

$$
\left\{\begin{array}{l}
-v^{\prime \prime}+\lambda_{1} v=h(t), 0<t<1, \\
v(0)=v(1)=0 .
\end{array}\right.
$$

So $v(t)=\int_{0}^{1} G_{1}(t, s) h(s) d s, t \in[0,1]$, namely

$$
-u^{\prime \prime}+\lambda_{2} u=\int_{0}^{1} G_{1}(t, s) h(s) d s, t \in[0,1] .
$$

Similarly, we get

$$
-u^{\prime \prime}+\lambda_{1} u=\int_{0}^{1} G_{2}(t, s) h(s) d s, t \in[0,1] .
$$

Owning to (1.7), (1.8) and lemma 1.2, for $\forall h \in Y$, we have

$$
\begin{aligned}
\left|u^{\prime \prime}(t)\right| & \leq\left|\lambda_{2}\right||u(t)|+\int_{0}^{1} G_{1}(t, s)|h(s)| d s \\
& \leq\left(\lambda_{2} \int_{0}^{1} \int_{0}^{1} G_{1}(t, s) G_{2}(s, \tau) d \tau d s+\int_{0}^{1} G_{1}(t, s) d s\right)\|h\|_{0} \\
& \leq\left(\left|\lambda_{2}\right| D_{1} D_{2}+D_{1}\right)\|h\|_{0} .
\end{aligned}
$$

Similarly to (1.9), by (1.6), (1.7) and lemma 1.2, we obtain

$$
\left|u^{\prime \prime}(t)\right| \leq\left(\left|\lambda_{1}\right| D_{1} D_{2}+D_{2}\right)\|h\|_{0} .
$$

Hence $\left\|u^{\prime \prime}(t)\right\|_{0} \leq M\|h\|_{0}$, in view of lemma 1.1, we get $\|T h\|_{1}=\|u\|_{1} \leq M\|h\|_{0}$, so $\|T\| \leq M$.

Let $K=\sup _{t \in[0,1]}[A(t)+B(t)-(\alpha+\beta)], g_{1}(t)=G_{1}(t, t), P=\left\{u \in Y_{+}\right.$: $\left.u(t) \geq \delta_{1} S(1-L) g_{1}(t)\|u\|_{0}, t \in[0,1]\right\}$, where if $\lambda_{i} \geq 0, S=1$, if $-\pi^{2}<\lambda_{i}<0$, $S=\sin \omega_{i}$, and assume

(A3) $L=K M<1$; 
$(A 4) \alpha<0, \beta>0$ or $\alpha \geq 0$.

Lemma 1.5. If $(A 1)-(A 4)$ hold, then $Q P \subset P$.

Proof. The proof for the conclusion of $\lambda_{i} \geq 0$ is completely similar and so we omit it. we only prove the result when $-\pi^{2}<\lambda_{i}<0$.

For $\forall h \in Y$, consider $\operatorname{BVP}(1.3)$ with $f=h$, obviously, it is equal to the following equation:

$$
\left\{\begin{array}{l}
u^{(4)}(t)+\beta u^{\prime \prime}(t)-\alpha u(t)=-(B(t)-\beta) u^{\prime \prime}(t)+(A(t)-\alpha) u(t)+h(t), \\
u(0)=u(1)=u^{\prime \prime}(0)=u^{\prime \prime}(1)=0
\end{array}\right.
$$

For $\forall v \in X$, setting $G v=-(B(t)-\beta) v^{\prime \prime}+(A(t)-\alpha) v$. It is easy to see that $G: X \rightarrow Y$ is linear and $|(G v)(t)| \leq[B(t)+A(t)-(\alpha+\beta)]\|v\|_{1} \leq K\|v\|_{1}$, so $\|G\| \leq K$. On the other hand, $u \in C^{2}[0,1] \cap C^{4}(0,1)$ is the solution of (1.11) if and only if $u \in X$ satisfies $u=T(G u+h)$, namely

$$
u \in X,(I-T G) u=T h .
$$

Owning to $G: X \rightarrow Y, T: Y \rightarrow X$, the operator $I-T G: X \rightarrow X$.Furthermore $\|T\| \leq M,\|G\| \leq K$ and $L=M K<1$ satisfy the conditions of the operator spectral theorem, so there exists $(I-T G)^{-1}$ which is bounded. If we set $H=$ $(I-T G)^{-1} T$ then (1.12) is equivalent to $u=H h$, by the Neumann expansion formula, we get

$$
H=\left(I+T G+\cdots+(T G)^{n}+\cdots\right) T=T+(T G) T+\cdots+(T G)^{n} T+\cdots .
$$

Since $T$ is completely continuous and $(I-T G)^{-1}$ is continuous, then $H$ is completely continuous. For $\forall h \in Y_{+}$, setting $u=T h$, then $u \in X \cap Y_{+}$and assuming $(A 4)$, then $u^{\prime \prime} \leq 0$, we have $(G v)(t)=-(B(t)-\beta) u^{\prime \prime}(t)+(A(t)-\alpha) u(t) \geq$ $0, t \in[0,1]$, i.e.

$$
\forall h \in Y_{+},(G T h)(t) \geq 0, t \in[0,1]
$$

By induction, for $\forall n \geq 1, h \in Y_{+}, t \in[0,1]$, we have $(T G)^{n}(T h)(t) \geq 0$. Hence, by (1.13) we get

$$
(H h)(t)=(T h)(t)+(T G)(T h)(t)+\cdots+(T G)^{n}(T h)(t)+\cdots T \geq(T h)(t) .
$$

So $H: Y \rightarrow Y_{+} \bigcap X$. On the other hand, $\forall h \in Y_{+}, t \in[0,1]$, we obtain

$$
\begin{aligned}
(H h)(t) & \leq(T h)(t)+\|T G\|(T h)(t)+\cdots+\|T G\|^{n}(T h)(t)+\cdots \\
& \leq\left(I+L+\cdots+L^{n}+\cdots\right)(T h)(t) \\
& =\frac{1}{1-L}(T h)(t) .
\end{aligned}
$$

So, the following inequalities hold:

$$
\|H h\|_{0} \leq \frac{1}{1-L}\|T h\|_{0} .
$$

For $\forall u \in P$, let $h=F u, Q=H F$, then $h \in Y_{+}$, by (1.15) we get

$$
(Q u)(t)=(H F u)(t) \geq(T F u)(t), t \in[0,1] .
$$


Owning to lemma $1.2, \forall t, \sigma \in[0,1]$, we have

$$
\begin{aligned}
(T F u)(t) & =\int_{0}^{1} \int_{0}^{1} G_{1}(t, s) G_{2}(s, \tau)(F u)(\tau) d \tau d s \\
& \geq \delta_{1} g_{1}(t) \int_{0}^{1} \int_{0}^{1} G_{1}(s, s) G_{2}(s, \tau)(F u)(\tau) d \tau d s \\
& \geq \delta_{1} g_{1}(t) \sin \omega_{i} \int_{0}^{1} \int_{0}^{1} G_{1}(\sigma, s) G_{2}(s, \tau)(F u)(\tau) d \tau d s \\
& \geq \delta_{1} g_{1}(t) \sin \omega_{i}(T F u)(\sigma) .
\end{aligned}
$$

So $(Q u)(t) \geq \delta_{1} g_{1}(t) \sin \omega_{i}\|T F u\|_{0}, t \in[0,1]$, by $(1.17)$, we get $\|T F u\|_{0} \geq(1-$ $L)\|H F u\|_{0}=(1-L)\|Q u\|_{0}$. Hence $(Q u)(t) \geq \delta_{1} g_{1}(t)(1-L) \sin \omega_{i}\|Q u\|_{0}$, i.e. $Q P \subset P$.

\section{MAin RESUlts}

We introduce the notations and assumptions as follows:

$$
\begin{gathered}
\bar{f}_{0}=\limsup _{u \rightarrow 0^{+}} \max _{t \in[0,1]} \sup _{v \in R} \frac{f(t, u, v)}{u}, \underline{f}_{0}=\liminf _{u \rightarrow 0^{+}} \min _{t \in[0,1]} \inf _{v \in R} \frac{f(t, u, v)}{u}, \\
\bar{f}_{\infty}=\limsup _{u \rightarrow+\infty} \max _{t \in[0,1]} \sup _{v \in R} \frac{f(t, u, v)}{u}, \underline{f}_{\infty}=\liminf _{u \rightarrow+\infty} \min _{t \in[0,1]} \inf _{v \in R} \frac{f(t, u, v)}{u}, \\
\Gamma=\pi^{4}-\beta \pi^{2}-\alpha, d_{1}=\min _{t \in\left[\frac{1}{4}, \frac{3}{4}\right]} g_{1}(t), \\
\delta=\delta_{1} S(1-L) d_{1}, b_{i}=\min _{\frac{1}{4} \leq t, s \leq \frac{3}{4}} G_{i}(t, s),
\end{gathered}
$$

where if $\lambda_{i} \geq 0, S=1$, if $-\pi^{2}<\lambda_{i}<0, S=\sin \omega_{i}$. It is easy to see that $\delta>0$ and $b_{i}>0$, the hypothesis $\frac{\alpha}{\pi^{4}}+\frac{\beta}{\pi^{2}}<1$ assures that $\Gamma>0$. We shall use the following assumptions:

(A5) There exist constants $p_{1}>0, a_{1} \geq 0, q_{1} \geq 0$ such that $f(t, u, v) \leq a_{1} u-$ $q_{1} v, \forall t \in[0,1], 0<u<p_{1},|v|<p_{1}$ and $a_{1}+q_{1} \pi^{2}<(1-L) \Gamma$

(A6) There exist constants $p_{2}>0, a_{2} \geq 0, q_{2} \geq 0$ such that $f(t, u, v) \geq a_{2} u+$ $q_{2}|v|, \forall t \in[0,1], 0<u<p_{2},|v|<p_{2}$ and $a_{2}-q_{2} \pi^{2}>\Gamma$.

Theorem 2.1. Assume that $\underline{f}_{\infty}>\Gamma, \underline{f}_{0}>\Gamma$, and $(A 1)-(A 5)$ hold then $B V P(1.3)$ has at least two positive solutions.

Proof. Let $\Omega_{p_{1}}=\left\{u \in P ;\|u\|_{0}<p_{1}\right\}$, for $\forall u \in \partial \Omega_{p_{1}}, 0<\mu \leq 1$, we get $\mu Q u \neq u$.

In fact, if $\exists u_{0} \in \partial \Omega_{p_{1}}$ and $0<\mu_{0} \leq 1$ such that $\mu_{0} Q u_{0}=u_{0}$ and (A4) hold, then $\lambda_{2}=\frac{-\beta-\sqrt{\beta^{2}+4 \alpha}}{2} \leq 0$, by $(1.7)$, we can get $u^{\prime \prime}(t) \leq 0, \forall t \in[0,1]$. Because (A5) we also have

$$
f\left(t, u_{0}, u_{0}^{\prime \prime}\right) \leq a_{1} u-q_{1} v, 0<u_{0}<p_{1},\left\|u_{0}^{\prime \prime}\right\|<p_{1}, \forall t \in[0,1] .
$$

By (1.16), we obtain $u_{0}=\mu_{0} Q u_{0} \leq Q u_{0} \leq \frac{1}{1-L}\left(T F u_{0}\right)$. Let $v_{0}=T F u_{0}$, then $u_{0} \leq \frac{1}{1-L} v_{0}$ and $v_{0}$ satisfies the $\operatorname{BVP}(1.4)$ with $h=F u_{0}$, i.e.

$$
v_{0}^{(4)}(t)+\beta v_{0}^{\prime \prime}(t)-\alpha v_{0}(t)=f\left(t, u_{0}(t), u_{0}^{\prime \prime}(t)\right) .
$$


Multiplying the above equation by $\sin \pi t$ and integrating on $[0,1]$ combined with $v_{0}(0)=v_{0}(1)=v_{0}^{\prime \prime}(0)=v_{0}^{\prime \prime}(1)=0$ and $(A 5)$, we get

$$
\begin{aligned}
\Gamma \int_{0}^{1} u_{0}(t) \sin \pi t d t & \leq \frac{1}{1-L} \Gamma \int_{0}^{1} \sin \pi t v_{0}(t) d t \\
& =\frac{1}{1-L} \int_{0}^{1} f\left(t, u_{0}(t), u_{0}^{\prime \prime}(t)\right) \sin \pi t d t \\
& \leq \frac{1}{1-L}\left(a_{1}+q_{1} \pi^{2}\right) \int_{0}^{1} u_{0}(t) \sin \pi t d t
\end{aligned}
$$

so $\Gamma<\frac{1}{1-L}\left(a_{1}+b_{1} \pi^{2}\right)$, which contradicts $a_{1}+q_{1} \pi^{2}<(1-L) \Gamma$. So $i\left(Q, \Omega_{p_{1}}, P\right)=1$.

By the definition of $\delta$ and $d_{1}$, we have

$$
\forall u \in P, u(t) \geq \delta\|u\|_{0}, t \in\left[\frac{1}{4}, \frac{3}{4}\right] .
$$

Owning to $\underline{f}_{0}>\Gamma$, we can choose $\varepsilon>0$ such that $\underline{f}_{0}>\Gamma+\varepsilon$, then there exists $0<r_{1}<p_{1}$ satisfies

$$
f(t, x, y)>(\Gamma+\varepsilon) x, t \in[0,1], 0<x \leq r_{1}, y \in R .
$$

Setting $\Omega_{r_{1}}=\left\{u \in P:\|u\|_{0}<r_{1}\right\}$, for any $u \in \partial \Omega_{r_{1}}$, we have $u(t) \geq \delta\|u\|_{0}=$ $\delta r_{1}, t \in\left[\frac{1}{4}, \frac{3}{4}\right]$, so

$$
f\left(t, u(t), u^{\prime \prime}(t)\right)>(\Gamma+\varepsilon) u(t) \geq(\Gamma+\varepsilon) \delta r_{1}, t \in\left[\frac{1}{4}, \frac{3}{4}\right] .
$$

Next we prove $(a)$ in $f_{u \in \partial \Omega_{r_{1}}}\|Q u\|_{0}>0,(b) \forall u \in \partial \Omega_{r_{1}}, 0<\mu \leq 1, Q u \neq \mu u$.

(a) $\forall u \in \partial \Omega_{r_{1}}$, by (1.14), we get

$$
\begin{aligned}
\|Q u\|_{0} \geq Q u\left(\frac{1}{2}\right) & \geq(T F u)\left(\frac{1}{2}\right)=\int_{0}^{1} \int_{0}^{1} G_{1}\left(\frac{1}{2}, s\right) G_{2}\left(\frac{1}{2}, \tau\right) f\left(\tau, u(\tau), u^{\prime \prime}(\tau)\right) d \tau d s \\
& \geq(\Gamma+\varepsilon) \delta r_{1} \int_{\frac{1}{4}}^{\frac{3}{4}} \int_{\frac{1}{4}}^{\frac{3}{4}} G_{1}\left(\frac{1}{2}, s\right) G_{2}(s, \tau) d \tau d s \\
& \geq \frac{1}{4}(\Gamma+\varepsilon) \delta b_{1} b_{2} r_{1} .
\end{aligned}
$$

So, we obtain in $f_{u \in \partial \Omega_{r_{1}}}\|Q u\|>0$.

(b) Assume on the contrary that $\exists u_{0} \in \partial \Omega_{r_{1}}$ and $0<\mu_{0} \leq 1$ such that $Q u_{0}=\mu_{0} u_{0}$. By $(1.15)$, we get $u_{0}(t) \geq \mu_{0} u_{0}(t)=\left(Q u_{0}\right) \geq\left(T F u_{0}\right)(t), t \in[0,1]$. Similarly to the proof of (2.1), we obtain

$$
\Gamma \int_{0}^{1} \sin \pi t u_{0}(t) d t \geq \int_{0}^{1} \sin \pi t f\left(t, u_{0}(t), u_{0}^{\prime \prime}(t)\right) d t
$$

By view of $f\left(t, u(t), u^{\prime \prime}(t)\right)>(\Gamma+\varepsilon) u_{0}(t)$, we have

$$
\Gamma \int_{0}^{1} \sin \pi t u_{0}(t) d t \geq(\Gamma+\varepsilon) \int_{0}^{1} \sin \pi t u_{0}(t) d t,
$$

so we get $\Gamma>\Gamma+\varepsilon$, this is a contradiction.

Now, owning to $(a)(b)$ and the fixed point index theory, we get $i\left(Q, \Omega r_{1}, P\right)=$ 0 . 
Because $\underline{f}_{\infty}>\Gamma$, we choose $\varepsilon>0$ such that $\underline{f}_{\infty}>\Gamma+\varepsilon$, then there exists $R_{0}>0$ satisfied with $f(t, x, y)>(\Gamma+\varepsilon) x, t \in[0,1], x>R_{0}, y \in R$. By $\sup _{(t, x, y) \in[0,1] \times\left[0, R_{0}\right] \times R} f(t, x, y)<\infty$, we know that $\exists M>0$ such that

$$
f(t, x, y)>(\Gamma+\varepsilon) x-M, t \in[0,1], 0<x \leq R_{1}, y \in R .
$$

Take $R_{1}>\max \left\{p_{1}, \delta^{-1} R_{0}, \frac{\sqrt{2} M}{\varepsilon \delta}\right\}$ and let $\Omega_{R_{1}}=\left\{u \in P:\|u\|_{0}<R_{1}\right\}$, next we prove (c) inf $f_{u \in \partial \Omega_{R_{1}}}\|Q u\|_{0}>0$ and $(d) \forall u \in \partial \Omega_{R_{1}}, 0<\mu \leq 1, Q u \neq \mu u$.

(c) Similar to $(2.2)$, we can get

$$
\begin{aligned}
\|Q u\|_{0} & \geq Q u\left(\frac{1}{2}\right) \geq(\operatorname{TFu})\left(\frac{1}{2}\right) \\
& \geq \int_{0}^{1} \int_{\frac{1}{4}}^{\frac{3}{4}} G_{1}\left(\frac{1}{2}, s\right) G_{2}(s, \tau) f\left(\tau, u(\tau), u^{\prime \prime}(\tau)\right) d \tau d s \\
& \geq \frac{1}{2}(\Gamma+\varepsilon) \delta b_{2} D_{1} R_{1} .
\end{aligned}
$$

Hence (c) inf $f_{u \in \partial \Omega_{R_{1}}}\|Q u\|_{0}>0$.

(d) Assume on the contrary that $\exists u_{0} \in \partial \Omega_{R_{1}}$ and $0<\mu_{0} \leq 1$ such that $Q u_{0}=\mu_{0} u_{0}$, by $(1.15)$ we have $\left(Q u_{0}\right)(t) \geq\left(T F u_{0}\right)(t), t \in[0,1]$. Similar to $(2.1)$ we have

$$
\begin{aligned}
\Gamma \int_{0}^{1} u_{0}(t) \sin \pi t d t & \geq \int_{0}^{1} f\left(t, u_{0}(t), u_{0}^{\prime \prime}(t)\right) \sin \pi t d t \\
& \geq(\Gamma+\varepsilon) \int_{0}^{1} u_{0}(t) \sin \pi t d t-M \int_{0}^{1} \sin \pi t d t
\end{aligned}
$$

so

$$
M \int_{0}^{1} \sin \pi t d t \geq \varepsilon \int_{0}^{1} u_{0}(t) \sin \pi t d t \geq \varepsilon \delta\left\|u_{0}\right\|_{0} \int_{\frac{1}{4}}^{\frac{3}{4}} \sin \pi t d t,
$$

thus $R_{1}=\|u\|_{0} \leq \frac{\sqrt{2} M}{\varepsilon \delta}$ which contradicts the choice of $R_{1}$. With the proof of (c) $(d)$, we get $i\left(Q, \Omega_{R_{1}}, P\right)=0$.

Hence

$$
\begin{gathered}
i\left(Q, \Omega_{R_{1}} \backslash \Omega_{p_{1}}, P\right)=i\left(Q, \Omega_{R_{1}}, P\right)-i\left(Q, \Omega_{p_{1}}, P\right)=0-1=-1, \\
i\left(Q, \Omega_{p_{1}} \backslash \Omega_{r_{1}}, P\right)=i\left(Q, \Omega_{p_{1}}, P\right)-i\left(Q, \Omega_{r_{1}}, P\right)=1-0=1 .
\end{gathered}
$$

Thus BVP(1.3) has at least two positive solutions $x_{1}, x_{2}$ such that $r_{1}<x_{1}<p_{1}<$ $x_{2}<R_{1}$.

Theorem 2.2. Assume that $\bar{f}_{\infty}<(1-L) \Gamma, \bar{f}_{0}<(1-L) \Gamma,(A 1)-(A 4)$ and (A6) hold, then BVP(1.3) has at least two positive solutions.

Proof. Set $\Omega_{p_{2}}=\left\{u \in P:\|u\|_{0}<p_{2}\right\}$, next we prove $(e) i n f_{u \in \partial \Omega_{p_{2}}}\|Q u\|_{0}>$ $0,(f) \forall u \in \partial \Omega_{p_{2}}, 0<\mu \leq 1, Q u \neq \mu u$. 
(e) $\forall u \in \partial \Omega_{p_{2}}$ by $(A 6)$ we have $f\left(t, u, u^{\prime \prime}\right) \geq a_{2} u+q_{2}\left|u^{\prime \prime}\right| \geq\left(a_{2}+q_{2}\right) p_{2}$, similar to (2.2), by lemma 1.2 we have

$$
\begin{aligned}
\|Q u\|_{0} \geq Q u\left(\frac{1}{2}\right) & \geq(T F u)\left(\frac{1}{2}\right)=\int_{0}^{1} \int_{0}^{1} G_{1}\left(\frac{1}{2}, s\right) G_{2}(s, \tau) f\left(\tau, u(\tau), u^{\prime \prime}(\tau)\right) d \tau d s \\
& \geq \int_{\frac{1}{4}}^{\frac{3}{4}} \int_{\frac{1}{4}}^{\frac{3}{4}} G_{2}(\tau, \tau) d \tau\left[a_{2}\|u\|_{0}+q_{2}\left\|u^{\prime \prime}\right\|_{0}\right] \\
& \geq \frac{1}{4} b_{1} b_{2}\left(a_{2}+q_{2}\right) p_{2},
\end{aligned}
$$

so $i n f_{u \in \partial \Omega_{p_{2}}}\|Q u\|_{0}>0$.

( $f$ ) Assume on the contrary that $\exists u_{0} \in \partial \Omega_{p_{2}}$, and $\mu_{0} \geq 1$ such that $\mu_{0} Q u_{0}=u_{0}$, in view of lemma $1.1,(A 6)$, and $u^{\prime \prime} \leq 0$ similar to $(2.1)$, we obtain

$$
\begin{aligned}
\Gamma \int_{0}^{1} u_{0}(t) \sin \pi t d t & =\int_{0}^{1} f\left(t, u_{0}(t), u_{0}^{\prime \prime}(t)\right) \sin \pi t d t \\
& \geq \int_{0}^{1}\left(a_{2} u_{0}(t)+q_{2}\left\|u^{\prime \prime}\right\|_{0}\right) \sin \pi t d t \\
& =a_{2} \int_{0}^{1} u_{0}(t) \sin \pi t d t-q_{2} \int_{0}^{1} u_{0}^{\prime \prime}(t) \sin \pi t d t \\
& =\left(a_{2}-q_{2} \pi^{2}\right) \int_{0}^{1} u_{0}(t) \sin \pi t d t .
\end{aligned}
$$

It is easy to see that it contradicts $a_{2}-q_{2} \pi^{2}>\Gamma$, so $i\left(Q, \Omega_{p_{2}}, P\right)=0$.

Owning to $\bar{f}_{\infty}<(1-L) \Gamma$, let $N=(1-L) \Gamma$, we choose $0<\varepsilon<N$ satisfied with $\bar{f}_{\infty}<N-\varepsilon$, so $\exists 0<r_{2}<p_{2}$ such that $f(t, x, y) \leq(N-\varepsilon) x, 0<x \leq r_{2}, 0 \leq$ $t \leq 1, y \in R$. Set $\Omega_{r_{2}}=\left\{u \in P:\|u\|_{0}<r_{2}\right\}$, then $\forall u \in \Omega_{r_{2}}, f\left(t, u(t), u^{\prime \prime}(t)\right)<$ $(N-\varepsilon) u(t)$. We shall prove $\forall u \in \partial \Omega_{r_{2}}, \mu \geq 1, Q u \neq \mu u$.

In fact, assume on the contrary that $\exists u_{0} \in \partial \Omega_{r_{2}}$ and $\mu_{0} \geq 1$ such that $Q u_{0}=$ $\mu_{0} u_{0}$, by (1.15) and setting $v_{0}=T F u_{0}$, similar to (2.1), we have

$$
\begin{aligned}
N \int_{0}^{1} u_{0}(t) \sin \pi t d t & \leq \Gamma \int_{0}^{1} v_{0}(t) \sin \pi t d t \\
& =\int_{0}^{1} f\left(t, u_{0}(t), u_{0}^{\prime \prime}(t)\right) \sin \pi t d t \\
& \leq(N-\varepsilon) \int_{0}^{1} u_{0}(t) \sin \pi t d t
\end{aligned}
$$

Because $\int_{0}^{1} u_{0}(t) \sin \pi t d t>0$, we get $N \leq N-\varepsilon$, which is a contradiction, $i\left(Q, \Omega_{r_{2}}, P\right)=1$.

By $\bar{f}_{0}<(1-L) \Gamma$, similar to the case of $\bar{f}_{\infty}<(1-L) \Gamma$, setting $N=(1-L) \Gamma$, we choose $0<\varepsilon<N$ such that $\bar{f}_{0}<(N-\varepsilon)$, then there exists $R_{0}>0$ for $x \geq R_{0}, f(t, x, y)<(N-\varepsilon) x, \forall t \in[0,1]$. Let $M=\sup _{(t, u, v) \in[0,1] \times[0, \infty) \times R} f(t, u, v)$, then

$$
f(t, x, y)<(N-\varepsilon) x+M, \forall t \in[0,1], x \in[0, \infty) .
$$


Take $R_{2}>\max \left\{p_{2}, R_{0}, \frac{\sqrt{2} M}{\varepsilon \delta}\right\}$ and let $\Omega_{R_{2}}=\left\{u \in P:\|u\|_{0}<R_{2}\right\}$. Next we shall prove $\forall u \in \partial \Omega_{R_{2}}, \mu \geq 1, Q u \neq \mu u$.

Given on the contrary, there exists $\mu_{0} \geq 1, u_{0} \in \partial \Omega_{R_{2}}$ satisfied with $Q u_{0}=$ $\mu_{0} u_{0}$. Similar to $(2.2)(2.4)$, we can get

$$
M \int_{0}^{1} \sin \pi t d t \geq \varepsilon \int_{0}^{1} u_{0}(t) \sin \pi t d t \geq \varepsilon \delta\left\|u_{0}\right\|_{0} \int_{\frac{1}{4}}^{\frac{3}{4}} \sin \pi t d t
$$

So $R_{2}=\|u\|_{0} \leq \frac{\sqrt{2} M}{\varepsilon \delta}$ which contradicts the choice of $R_{2}$. Hence, by the fixed point index theory, we get $i\left(Q, \Omega_{R_{2}}, P\right)=1$.

So

$$
\begin{aligned}
& i\left(Q, \Omega_{R_{2}} \backslash \Omega_{p_{2}}, P\right)=i\left(Q, \Omega_{R_{2}}, P\right)-i\left(Q, \Omega_{p_{2}}, P\right)=1-0=1, \\
& i\left(Q, \Omega_{p_{2}} \backslash \Omega_{r_{2}}, P\right)=i\left(Q, \Omega_{p_{2}}, P\right)-i\left(Q, \Omega_{r_{2}}, P\right)=0-1=-1,
\end{aligned}
$$

namely, $\operatorname{BVP}(1.3)$ has at least two positive solutions $x_{1}, x_{2}$ such that $r_{2}<x_{1}<$ $p_{2}<x_{2}<R_{2}$.

Corollary 2.3. Assume that $(A 1)-(A 4)$ hold and either

(i) $\underline{f}_{0}>\Gamma, \bar{f}_{\infty}<(1-L) \Gamma$; or

(ii) $\overline{\bar{f}}_{0}<(1-L) \Gamma, \underline{f}_{\infty}>\Gamma$;

then BVP(1.3) has at least one solution.

\section{REFERENCES}

1. R.P. Agarwal, On fourth-order boundary value problems arising in beam analysis, Diff. Inte. Eqns. 2 (1989), 91-110.

2. Z.B. Bai, The method of lower and upper solution for a bending of an elastic beam equation, J. Math. Anal. Appl. 248 (2000), 195-202.

3. Z.B. Bai, Positive solutions for some second-order four-point boundary value problems, $J$. Math. Anal. Appl. 2 330(2007), 34-50.

4. Z.B. Bai, The method of lower and upper solutions for some fourth-order boundary value problems, Nonlinear Anal. 67 (2007), 1704-1709.

5. G.Q. Chai, Existence of positive solutions for fourth-order boundary value problem with variable parameters, Nonlinear Anal. 66 (2007), 870-880.

6. D. Guo, V. Lakshmikantham, Nonlinear Problems in Abstract Cones, Academic Press, New york 1988.

7. Y.X. Li. Existence and method of lower and upper solutions for fourth-order nonlinear boundary value problems, Acta Mathmatic Scientia 23 (2003),245-252.

8. Y.X. Li, Existence and multiplicity of positive solutions for fourth-order boundary value problems, Acta Mathematicae Applicatae Sinica 26 (2003), 109-116.

9. Y.X. Li, Positive solutions of fourth-order boundary value problems with two parameters, J. Math. Anal. Appl. 281 (2003), 477-484.

10. R.Y. Ma and H.Y. Wang, Positive solutionS of nonlinear three-point boundary-value problems, Nonlinear Anal. 279 (2003), 216-227.

11. Z.L. Wei and C.C. Pang, Positive solutions and multiplicity of fourth-order m-point boundary value problem with two parameters, Nonlinear Anal. 67 (2007), 1586-1598. 
1 College of Information Science and Engineering,Shandong University of Science And Technology, Qing Dao, 266510, P. R. China.

E-mail address: xiaoxin7716@126.com

${ }^{2}$ College of Information Science and Engineering,Shandong University of Science And Technology, Qing Dao, 266510, P. R. China.;

AMIRKABIR UNIVERSITY

E-mail address: zhanbingbai@163.com 